CASE REPORT | STOMACH

\title{
Iron Pill-Induced Gastritis
}

\author{
Jana G. Hashash, MD¹, Siobhan Proksell, MD², Shih-Fan Kuan, MD³, and Jaideep \\ Behari, MD, $\mathrm{PhD}^{1}$ \\ ${ }^{1}$ Division of Gastroenterology, Hepatology, and Nutrition, University of Pittsburgh Medical Center, Pittsburgh, PA \\ ${ }^{2}$ Department of Medicine, University of Pittsburgh Medical Center, Pittsburgh, PA \\ ${ }^{3}$ Department of Pathology, University of Pittsburgh Medical Center, Pittsburgh, PA
}

\begin{abstract}
Iron-deficiency anemia is a prevalent condition treated with iron supplementation. Iron pill-induced gastritis is an under-recognized, albeit serious potential complication of iron pill ingestion. This entity must be identified by healthcare providers who prescribe iron. We present a case of a 59-year-old male with iron deficiency anemia on ferrous sulfate tablets who underwent an upper endoscopy, during which a single superficial gastric ulceration in the body was noted. Biopsies revealed heavy iron deposition confirming the ulceration was a consequence of the iron tablets. Iron pill-induced gastritis causes corrosive mucosal injury similar to that caused by chemical burns.
\end{abstract}

\section{Introduction}

Oral iron supplementation, usually in the form of a pill or tablet, is the most common treatment of iron-deficiency anemia. A potential complication of using oral iron supplementation is iron pill-induced gastritis. All gastroenterologists should be aware of the endoscopic findings associated with iron pill-induced gastritis, because they play a crucial role in diagnosing this condition. Patients with iron pill-induced gastritis should have their iron pills or tablets replaced by liquid iron. It is thought that iron pills and tablets cause a corrosive mucosal injury and that the effect is concentration-dependant. For this reason, liquid iron does not have the same side effect of promoting mucosal injury.

\section{Case Report}

A 59-year-old male with Child-Pugh B cirrhosis from nonalcoholic fatty liver disease and prior history of esophageal variceal bleeding presented to our gastroenterology suite for an upper gastrointestinal endoscopy for prophylactic variceal band ligation. The patient also had diabetes mellitus, hypertension, dyslipidemia, and chronic kidney disease (serum creatinine $1.4 \mathrm{mg} / \mathrm{dL}$ and a glomerular filtration rate $52 \mathrm{~mL} / \mathrm{min} / 1.73 \mathrm{~m}^{2}$ ). Among his medications were metformin, insulin glargine, ferrous sulfate, fenofibrate, and valsartan. The patient was receiving $325 \mathrm{mg}$ of ferrous sulfate by mouth twice daily. The patient appeared comfortable and had no complaints. His vital signs were within normal limits and his physical examination was significant for splenomegaly with spleen tip palpable $5 \mathrm{~cm}$ below the costal margin. Blood work revealed hemoglobin $11.4 \mathrm{~g} / \mathrm{dL}$ (normal: $14-18$ $\mathrm{g} / \mathrm{dL}$ ), a normal mean corpuscular volume of $91 \mathrm{fL}$, iron saturation 14\% (normal: 25-50\%), ferritin $88 \mathrm{ng} / \mathrm{mL}$ (normal: 10-282 ng/mL), and total iron binding capacity $339 \mu / \mathrm{dL}$ (normal: 250-420 $\mu / \mathrm{dL}$ ).

On upper endoscopy, the patient was found to have esophageal varices in the distal esophagus and mild portal hypertensive gastropathy. One superficial 6-mm gastric ulcer was found in the gastric body (Figures 1 and 2). Biopsies from this ulcer revealed erosive gastritis. An iron stain demonstrated heavy iron deposition consistent

ACG Case Rep J 2013;1(1):13-15. doi:10.14309/crj.2013.7. Published online: October 8, 2013.

Correspondence: Jana G. Hashash, MD, Division of Gastroenterology, Hepatology, and Nutrition, University of Pittsburgh Medical Center, 200 Lothrop Street PUH, M2, C Wing, Pittsburgh, PA 15213 (alhashashj@upmc.edu)

Copyright: () 2013 Hashash et al. This is an open-access article distributed under the terms of the Creative Commons Attribution License, which permits unrestricted use, distribution, and reproduction in any medium, provided the original author and source are credited. 
with iron pill-induced gastritis (Figures 3 and 4). The patient was started on an oral proton pump inhibitor and instructed to discontinue his oral iron tablets. He was started on a liquid iron preparation instead of the iron tablets. Of note, the patient had a liver biopsy performed a few years prior to this presentation at an outside hospital, as part of his liver cirrhosis work-up, and this did not show any evidence of iron overload.

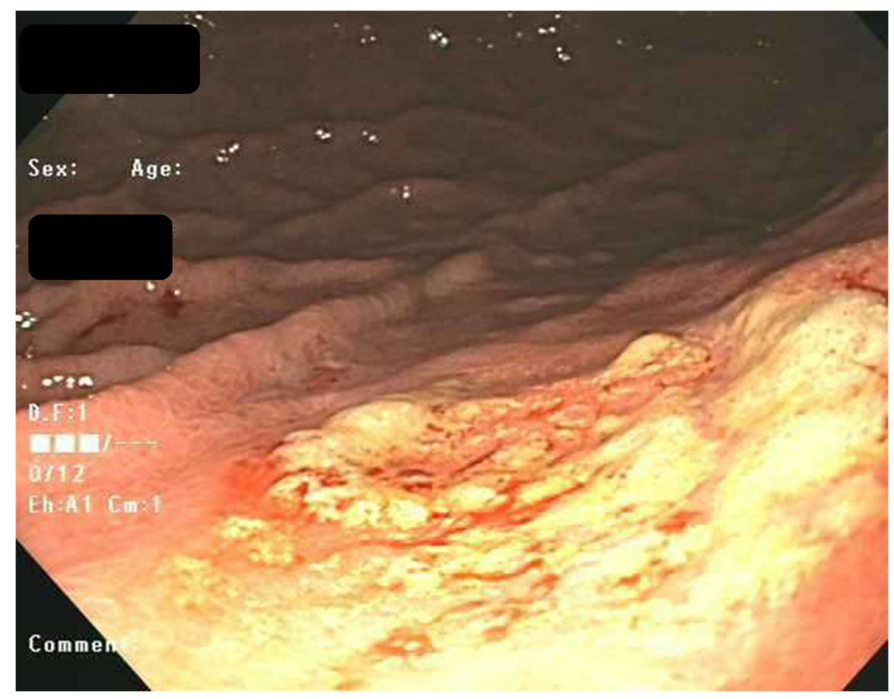

Figure 1. Endoscopic appearance of the gastric ulcer margins.

\section{Discussion}

According to the most recent data published by the World Health Organization, almost 25\% of the world's population carries a diagnosis of anemia, the majority being of the iron deficiency type. ${ }^{1}$ Ferrous sulfate supplementation in the pill or tablet form is a commonly prescribed treatment for these patients with iron deficiency anemia. Common side effects of oral iron supplementation include constipation,

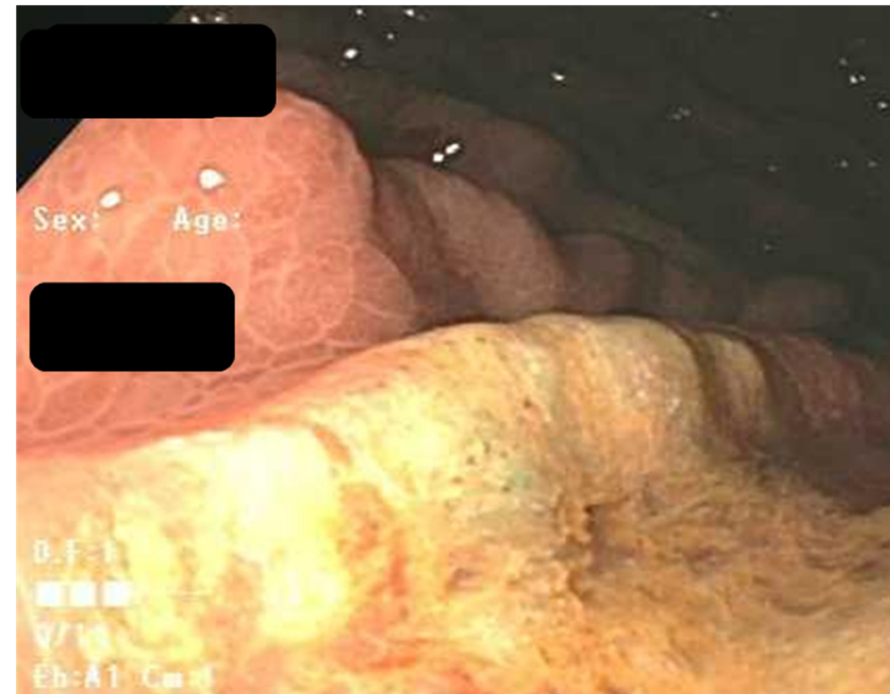

Figure 2. Endoscopic appearance of the gastric ulcer bed.

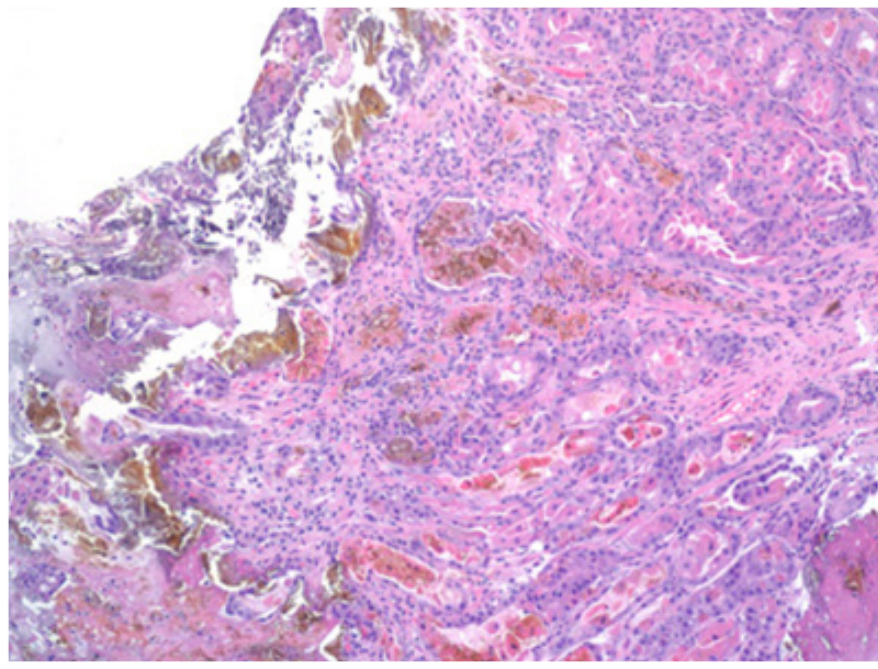

Figure 3. Hematoxylin and eosin stain of the gastric ulcer at 100 magnification.

dark stools, and gastrointestinal irritation, often in the form of nausea. ${ }^{2}$ Iron pill gastritis is a documented complication of iron supplementation, but is rarely encountered. Recently, however, the topic has been garnering increasing attention. Kaye et al published a prospective study investigating the effects of iron supplementation on patients with iron deficiency anemia. ${ }^{3}$ Of 16 patients with iron deposition confirmed by biopsy, 6 had visible erosions on endoscopy, a significantly greater proportion than those patients without iron supple-

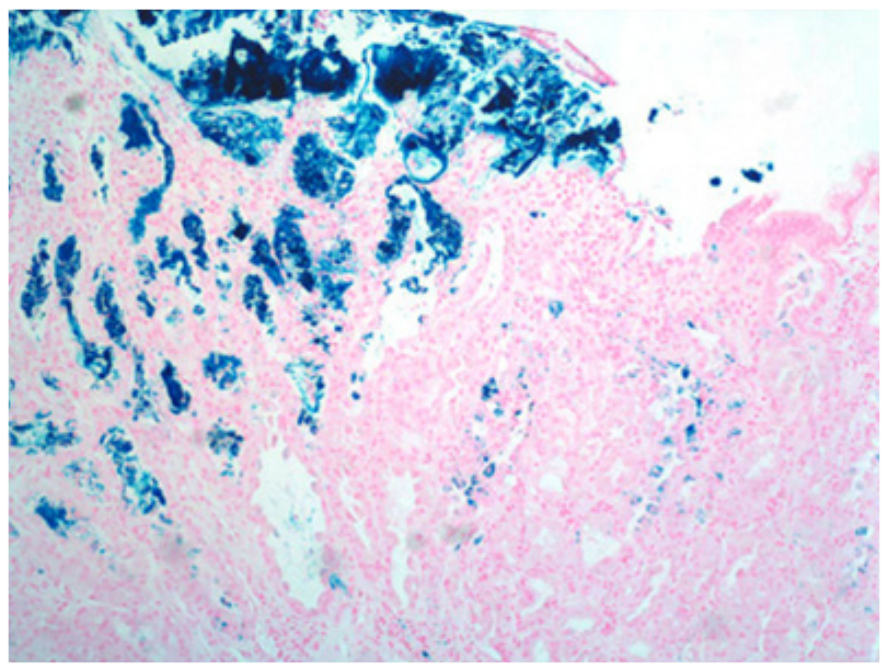

Figure 4. Iron stain of the gastric ulcer revealing heavy iron deposition at 100 magnification.

mentation (14/141). No significant differences were attributed to potential confounders such as age, gender, aspirin and non-steroidal anti-inflammatory drug use. Kaye et al also included a retrospective analysis reviewing the cases of 59 patients with iron deposition, 58 of whom were receiving oral iron. Of this population, 31 were documented to have erosions and ulcerations by endoscopy report. 
Laine et al published a prospective evaluation of healthy volunteers who were separated into 2 groups, both of which had not had previous iron supplementation. ${ }^{4}$ The first group was assigned to receive ferrous sulfate $325 \mathrm{mg}$ orally 3 times daily for a duration of 2 weeks, while the second group took the supplement at the same daily dose and frequency for only 1 week. Endoscopy was performed prior to and after the completion of iron therapy and showed a significant increase in endoscopic abnormalities of the stomach in patients who received iron therapy for a longer duration. Two patients had developed gastric ulcerations after iron therapy.

Iron pill-induced gastritis is an under-recognized, albeit serious entity that merits further investigation. Many patients who are diagnosed with iron pill gastritis have numerous comorbidities that may increase their susceptibility to this condition, though substantial investigation into this subject has not been undertaken. Iron has been noted to cause a focal erosive mucosal injury similar to that caused by a chemical burn. Endoscopically, this manifests as an erosion, an ulceration, or diffuse gastritis. Iron deposits a brown-black crystalline hemosiderin that erodes the mucosa. It is thought that iron erodes the mucosa through a direct corrosive effect that subsequently produces a local injury to the mucosa in a concentration-dependant manner. In some patients, particularly these with other comorbid conditions such as hemochromatosis, gastric antral vascular ectasia, and gastric adenocarcinoma, among others, the degree of iron deposition extends to the lamina propria and even the gastric glands. Iron pillinduced gastritis only occurs when iron supplementation is given in the tablet or pill form likely because of the concentration effect, but has not been noted to occur in patients who are placed on a liquid form of iron supplementation. This case report is intended to remind gastroenterologists of the possible risks of iron pill supplementation.

\section{Disclosures}

Author contributions: J.G. Hashash: study concept and design, acquisition of data, analysis and interpretation of data, drafting of the manuscript; S. Proksell: acquisition of data, drafting of the manuscript; S.F. Kuan: aquisition of data, analysis and interpretation of data; J. Behari: study concept and design, acquisition of data, analysis and interpretation of data, study supervision. J.G. Hashash is the article guarantor.

Financial disclosure: No conflicts of interest or sources of funding exist.

Received: June 9, 2013; Accepted: September 10, 2013

\section{References}

1. World Health Organization. Vitamin and Mineral Nutrition Information System. Worldwide prevalence on anaemia 1993-2005. World Health Organization web site. http://www.who.int/vmnis/database/anaemia/ anaemia_status_summary/en/index.html. Accessed June 6, 2013.

2. Ferrous sulfate: Patient drug information. UpToDate web site. http:// www.uptodate.com/contents/ferrous-sulfate-patient-drug-information. Accessed June 6, 2013

3. Kaye P, Abdulla K, Wood J, et al. Iron-induced mucosal pathology of the upper gastrointestinal tract: a common finding in patients on oral iron therapy. Histopathology. 2008;53(3):311-7.

4. Laine LA, Bentley E, Chandrasoma P. Effect of oral iron therapy on the upper gastrointestinal tract. A prospective evaluation. Dig Dis Sci. 1998;33(2):172-7.

\section{Publish your work in ACG Case Reports Journal}

ACG Case Reports Journal is a peer-reviewed, open-access publication that provides Gl fellows, private practice clinicians, and other members of the health care team an opportunity to share interesting case reports with their peers and with leaders in the field. Visit http://acgcasereports.gi.org for submission guidelines. Submit your manuscript online at http://mc.manuscriptcentral.com/acgcr. 